Original Research Paper

\title{
Survey Report on Sending Data Securely using IoT Over Cloud
}

\author{
Om Prakash Singh ${ }^{1}$, Ranjith CS ${ }^{1}$, Karthik R. ${ }^{1}$, Sandeep Reddy ${ }^{1}, \operatorname{Rashmi~Soni~}^{1}$ \\ ${ }^{1}$ Department of Information Science Engineering, Dayananda Sagar Academy of \\ Technology and Management, Bengaluru, India.
}

Article history

Received:

25.02.2020

Revised:

10.03.2020

Accepted:

16.03.2020

*Corresponding Author:

Gagan Deep Shivamadhu

Email:

drrashmicse@gmail.com

This is an open access article, licensed under: $C C-B Y-S A$
Abstract: In recent times, everything around us is automated. People are looking to make things easier and easier day by day, by reducing the work they do, but not reduce the efficiency of work. The most important thing in this technically forward world, is, what we mentioned above i.e. Automation and Security. Travel needs a lot of automation in this day and age, especially Airports. The idea of a bag can check-in by itself, and tell us where it is and deprive us of the worry of continuously checking or thinking if the luggage is okay, is just a dream for most people.

Keyword: Cloud Data, Cloud Security, Data Privacy, Data Security. 


\section{Introduction}

The Internet of Things (IoT) refers to the type of network that links things, i.e. physical object the vices, homes, vehicles and other items embedde $\mathrm{d}$ in protocol based software, sensors and network connectivit y which allows data collection and sharing of the se objects.

In recent years, a lot of research has focused on data mining on the Internet of Things that connects Clustering can be defined as the procedure where in the set of abstract objects is made into class of indistinguishable object.

In recent years, on the Internet of Things, a lot of research has centered on data mining which links physical objects, people to people, people to machines or machines via the internet to machines.

As of late, due to a wide range of sensor gadgets on layer detection, a growing number of applications handle countless sensor data in the Internet of Things (IoT).

The comprehensive scaling of heterogeneous se nsors produces an issue of information International Journal of Scientific Research in Science, Engineering and Technology, which is one of the key issues for the implementation of the IoT model.

Sensors in IoT applications sense the complicat ed environment and generate huge data that need $\mathrm{s}$ to be filtered and cleaned so that it can be inter preted and the data collected in the form of patte rns will be provided to the user [1].

IoT enables object sensing and remote access ac ross different network infrastructures, which in turn allows for better integration between the real and computerized world. This results in improved efficiency, reliability and improved economic results. That request can be exceptionally recognized by using its configured registration system, but these entities can interoperate within the Internet's c urrent infrastructure. We refer to it as Data Mining as the way to find and investigate useful trends in a vast amount of information.

The IoT environment leads to a new era of engin eering and interaction in which entities can inter act, calculate and turn the data according to the $r$ equirements.

\section{Literature Review}

\subsection{Sensors: Ultrasonic Range Finder}

A sensor is a transducer system capable of detect ing events or changes in its environment and the $n$ producing an appropriate electrical output. A s ensor's most important features are precision, res olution, linearity, and speed. Calibration of the s ensor increases the performance of the sensor.

Ultrasonic range finders measure distance by emitting a pulse of ultrasonic sound that travels through the air until it hits an object. When that pulse of sound hits an object, it's reflected off the object and travels back to the ultrasonic range finder.

Ultrasonic range finders measure distance by em itting anultrasonic sound pulse that travels throu gh the air until an object is struck. As shown in Figure 1.

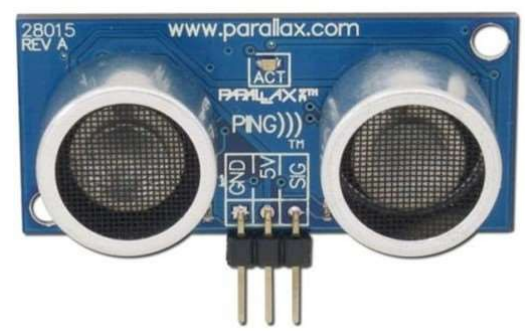

Figure 1. Ultrasonic Range Finder

\subsection{Cryptographic Algorithm}

That ensures the key for encryption is equivalent to the key for decryption. Figure is the simplifie d template of technique of conversional encrypti on. There are usually two types of symmetric cip hers, stream ciphers and block ciphers.

There are various cryptographic algorithms avail able based on traditional key distribution crypto graphy as modern cryptography is called symme tric encryption or single key encryption. 


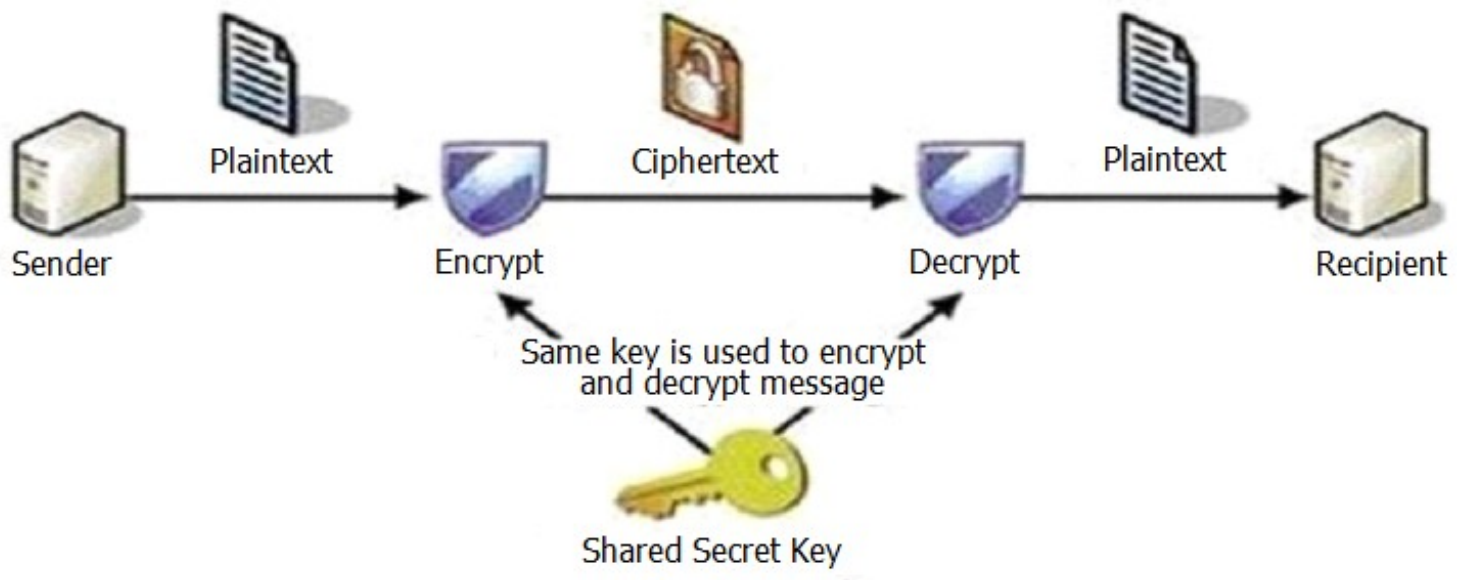

Figure 2. Symmetric Encryption Technique [1]

\section{Architectural Elements of IoT}

Embedded systems play a very important role in the creation and design of the IoT world. In gene ral, there are four main components in the IoT process. TheInternet is the first and most important thing. The second important thing is a device ca pable of transmitting real-time data across the network

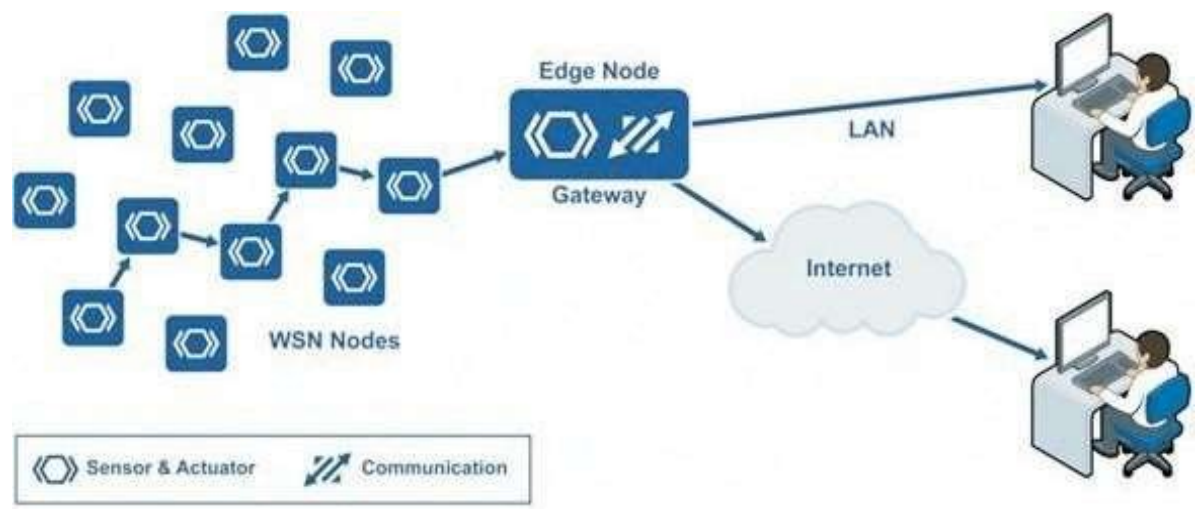

Figure 3. General IoT Architecture

\section{The Proposed Architecture to Maintain the Security Over the IoT Data}

Use four types of interconnected systems such as objects, gateways, network and cloud, IoT architecture can be represented.

IoT software can be used in different fields or or ganizations, but the main problem is information protection

Proposed architecture can be seen in Figure 4.

\section{AES-GCM}

Only the AES encryption is used in the AES- GCM with 128 bit input and output blocks. The main size could be calculated on the basis of the safety requirements as AES128 (with 10 rounds), AES192 (with 12 rounds) or AES- 256 (with 14 rounds). 


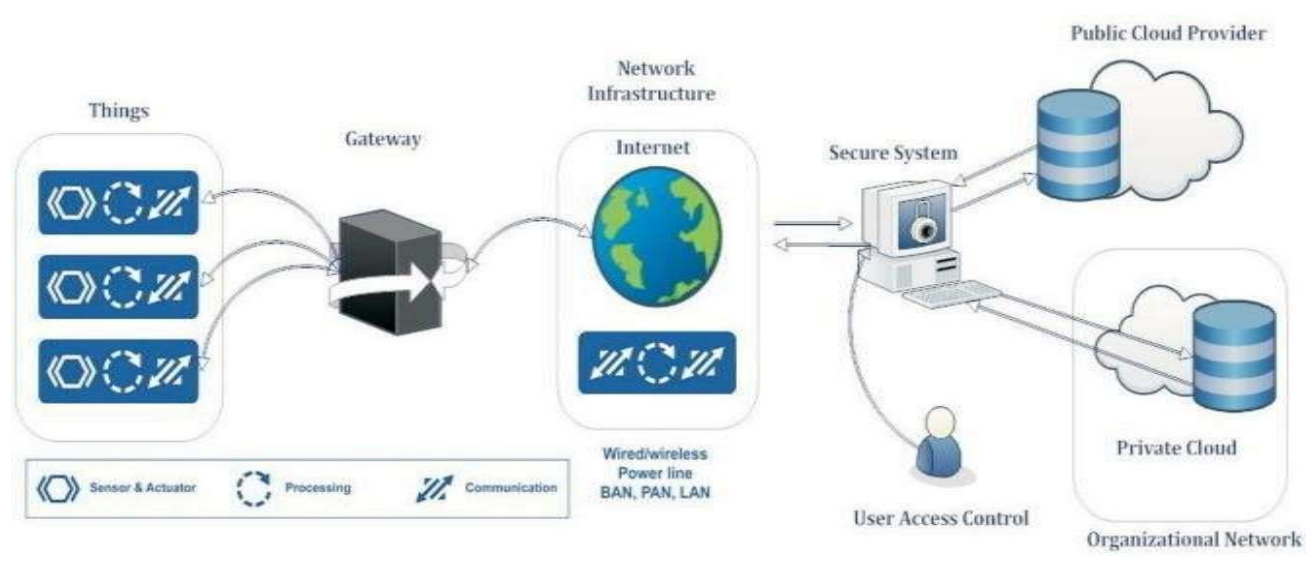

Figure 4. Proposed Architecture

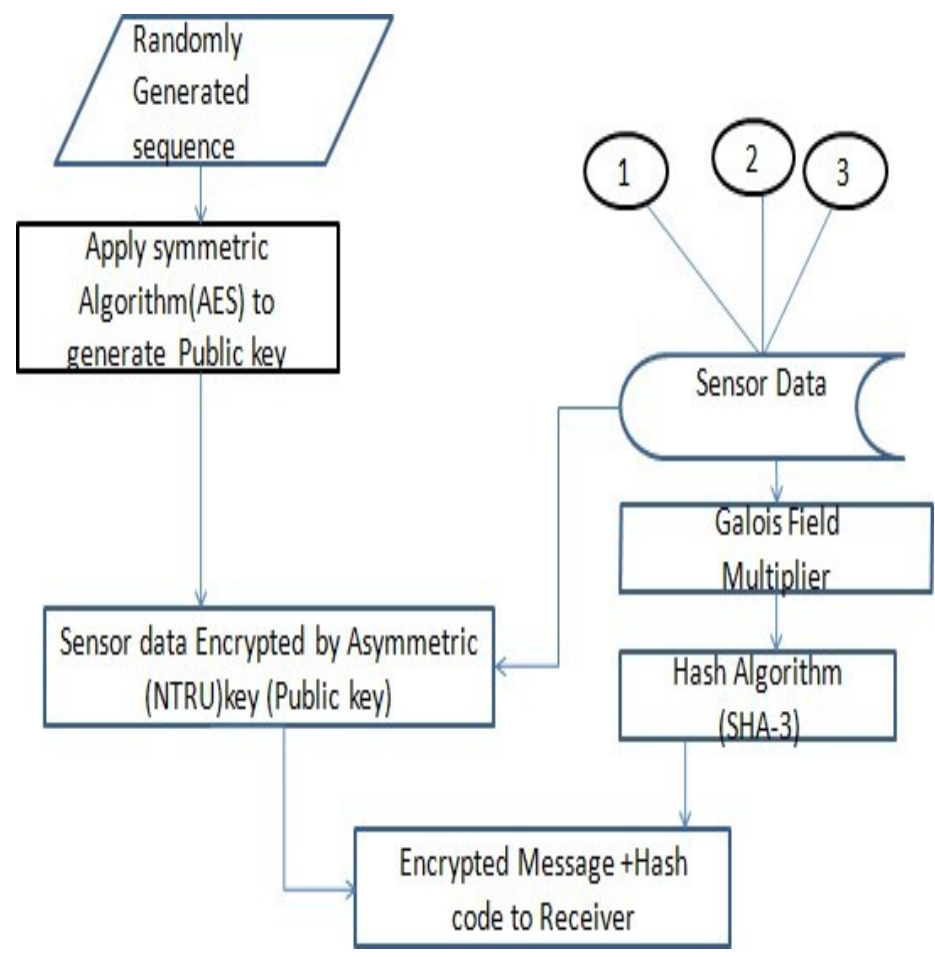

Figure 5. Proposed System Encryption Process

It is the responsibility of the cloud service provider to administer, maintain and update cloud hardwa re resources to client requirements. It is also the du ty of the backbone layer to distribute hardware resources efficiently, quickly and smooth. 


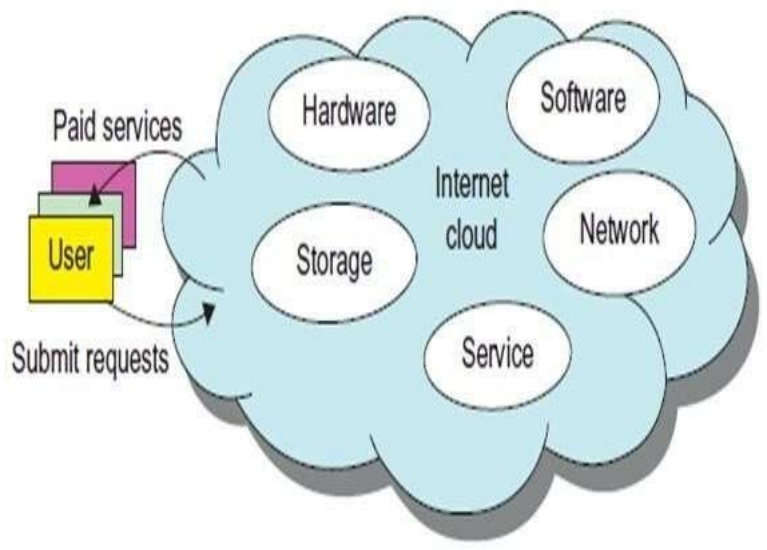

Figure 6. Cloud Environment

\section{Previous work}

There are some work related to this research:

- Trivedi \& Raval [1] represents the speed of different algorithms as the result of the hybrid encryption algorithm will be better and faster.

- Naeini [2] present the results of their interview study and following the flow of the interview. They discuss interviewees' IoT device purchase behaviors and the evaluation of our privacy and security label

- Wadhwani et al [3] provide and demonstrate the strength of IoT capable of contributing the services for the purpose of building vast number of applications and helping them to be implemented on the public platform.

- Bokefode et al [4] tackles the security problem in a cloud storage environment

- Kanade [5] place sensitive data safely on Organic Optical Data Store.

- Bhise et al [6] proposed a device, in a cloud storage environment and it solves the security issue.

- Karthik et al [7] proposed a designing monitoring systems is to reduce the cost of health care by minimizing appointments, hospitalizations and diagnostic testing procedures to doctor offices.

- Sharma et al [8] propose a project is to collect leaked gas around the gas sensor after sensing if the sensor value exceeds the threshold value and the microcontroller performs its programmed tasks.

- Mourvika et al [9] said that the Internet of Things (IoT) and its applications are becoming part of our daily lives, working methods and industry. There is much work on the design of key building blocks and models for next-generation Internet services enabled by a variety of connected things.

- Anjali et al [10] introduced the IoT based smart garbage monitoring system using ESP8266 with the GPS link. It will provide the improved efficient solution to the waste management issue over the previous systems.

\section{References}

[1] D. M. Trivedi, and T. J. Raval, "AES-GCM, NTRU public key cryptographic algorithm," IEEE, 2016.

[2] P. E. Naeini, "Semi-Structured Interview Study, Follow-Up Survey," IEEE, 2016.

[3] S. Wadhwani, U. Singh, P. Singh, and S. Dwivedi, "Ldr \& Wi-Fi Module, The IoT facilitates numerous benefits for society," IEEE, 2017.

[4] J. B. Bokefode, S. A. Ubale, and R. M. Gaikwad, "Range of Spectrum medium of transferring data - data transfer speed," IEEE, 2018. 
[5] V. A. Kanade and F. Nails, "Lens of the Eye, Blood Nano Infusion," IEEE, 2017.

[6] A. S. Bhise, A. Prajakta, S. Dattatray, and G. Modani, "Things, Gateways, Network Infrastructure," IEEE, 2016.

[7] B. N. Karthik, L. D. Parameswari, R. Harshini, and A. Akshaya, "Designing Monitoring Systems Is To Reduce The Cost Of Health Care," IEEE, 2018.

[8] M. SHarma, D. Tripathi, N. P. Yadav, and P. Rastogi, "Piccolo MCU, MQ-6 Sensors," IEEE, 2018.

[9] M. Shirode, M. Adaling, and J. Biradar, "Sensors and Working with Thing Speak," IEEE, 2018.

[10] A. V. Tarone, A. Katgube, and H. H. Shendre, "Cloud, GPS, ID, IR sensor, ESP8266, and ATMEGA328," IEEE, 2018. 